The $25^{\text {th }}$ International Symposium on Automation and Robotics in Construction

June 26-29, 2008

ISARC-2008

\title{
HYBRID ADVISORY SYSTEMS AND THE POSSIBILITIES OF IT USAGE IN THE PROCESS OF INDUSTRIAL FLOORING REPAIRS
}

\author{
Marcin Gajzler \\ Assistant \\ Poznan University of Technology \\ Institute of Structural Engineering \\ PL 60-965 Poznan, Piotrowo 5 \\ marcin.gajzler@ikb.poznan.pl
}

\begin{abstract}
It is very often the case that the units responsible for the maintenance of the building have to repair industrial flooring. The large number of available materials and technologies as well as some other factors, make it necessary to use tools which would help to make good decisions. Hybrid advisory system is a tool which supports the process of decision making and is responsible for generating possible solutions and related technological and economical information. From the point of view of the decision-maker such information is necessary in order to make the right choices. The system generates answers based on the input vector which accounts for factors such as the type of the damage, its size, the time during which the floor was affected by various influences and the possibilities of reparation. Emphasized hybridity refers to the fact that within one system there are numerous intelligent tools connected to the individual system modules. There are also some aspects of „methodological" hybridity, which is represented by the two ways of approaching the methods of knowledge acquisition in the system.
\end{abstract}

\section{KEYWORDS}

Advisory systems, hybrid model, industry flooring repairs

\section{INTRODUCTION}

Advisory systems are specialized and developed form of already known expert systems reckoned as intelligent. Their usage is becoming more and more common also in construction industry $[1,2]$. One of the suggested uses of advisory system is support in decision taking within the scope of material and technological solutions for the sake of repairing damaged industrial floor. The complexity of engineering problem and large number of possible solu- 
tions cause the situation in which decision taking within the scope of that problem requires the support of an intelligent and automated tool. Additional argument that advocates such solution is repeatability of the problem from the point of view of potential users - technical staff responsible for maintenance of industrial structures (large-surface logistic and trade centres, industrial shops), with additional frequency of problem occurrence.

\section{ADVISORY SYSTEM IDEA}

During the process of the analysis, a solution for decision taking problem, which occurs during realization of engineering problem - repairing the damaged concrete industrial floor, was sought. The analysis showed specific stages of decision taking problem with characteristics of taken decisions from strategic decisions to operational ones. One of the final stages of decision taking process is taking the decision on the selection of material and technological solution predestined in particular technical and non-technical conditions, connected with the damage, object and decision-maker's preferences. Due to large number of possible decision-related solutions and to problem's complexity, the concept of decision taking support was sought at this stage. The suggested solution turned out to be an advisory system that realizes the premises of last stage of decision taking and generates answers within the scope of possible solutions, technological information and economic parameters. Basing on that information, the decision maker takes the final decisions intended for implementation.

The proposed advisory system (Fig. 1) consists of knowledge base (KB), database (DB), inference engine (IE), dialogue interface (DI), update mechanisms and tool modules responsible for specialized functions in the system. Tool modules used in the systems include:

- modules that make use of characteristics of fuzzy sets (FS), which are responsible for information flow and its compatibility in relation to other elements of the system,

- artificial neural networks (ANN) as a tool for inference management and that functions within the inference engine.

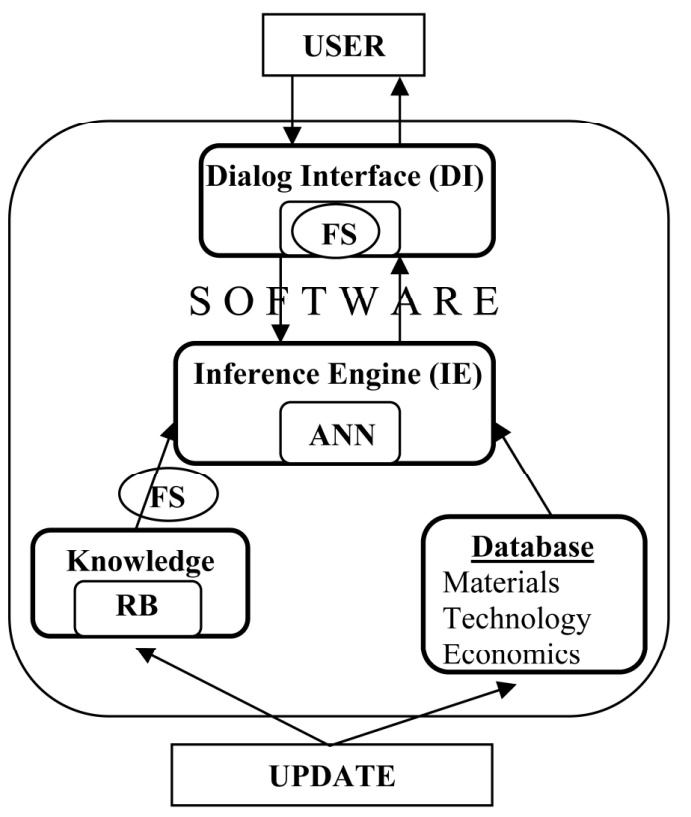

Figure 1. Hybrid advisory system model

From the point of view of reciprocal unification of intelligent techniques (fuzzy sets, artificial neural networks) in one intelligent tool - advisory system, system's character may be described as hybrid. Examples of other hybrid models one can find in [3-5].

\section{FUZZY KNOWLEDGE BASE}

One of most fundamental and important elements of presented advisory system is the knowledge base, which is reckoned as system resources. In this particular solution the decision was taken to obtain and record knowledge in form of rules of "if ..., then ..." type. This decision resulted form "friendly" and simple form of such record. Knowledge recorded in form of the rules was the result of knowledge acquisition session [6] with the participation of knowledge-field expert that took place during personal interview supported by the use of paper formular. The fact that the expert used natural and linguistic concepts of fuzzy character caused some difficulties for the system engineer. Therefore, the base was of fuzzy character, apart from being rule-based. That fact resulted from the intended laxity during the time 
of knowledge acquisition, and in turn created opportunity to eliminate sharp values, which could soon become outdated (particularly within the range of technical parameters). Implementation of fuzzy concepts gave some opportunities within the scope of knowledge updating and modification by the use of modification of membership functions related to specific fuzzy concepts. The same expert, who participated in knowledge acquisition, defined fuzzy concepts and membership functions. As a result, the element of the expert's mental model was coded in the knowledge base.

The created knowledge base was not fully completed and initially it contained 164 fuzzy rules. Noncomplementariness of the knowledge base was purposeful due to later knowledge's functioning in artificial neural networks connected with inference engine. In the case of knowledge base, both the complementariness was checked within the scope of the rules (premises and conclusions) and possible contradictions within the rules (ambiguous rules, excluding and duplicating rules). Each rule's construction contains 7 premises (features) and 5 conclusions (each corresponding to specific solutions). The content of the knowledge base reduced originally to 5 material and technological solutions, and within their scope it is possible to repair the damaged industrial floor. Limitation to these 5 solutions was the result of the time limitation of knowledge acquisition only. Depending on the need to develop the system there is the possibility to update and build up the knowledge base with the knowledge connected with new solutions and simultaneously to build up the database. These actions were to constitute an update in the system.

\section{INFERENCE ENGINE}

The inference engine is the "heart" of the system apart from its resources (data and knowledge). Various inference techniques (backward-, forwardchaining) unified with the knowledge base function there. As an alternative for these techniques and due to the non-complementariness of knowledge base it was assumed to have artificial neural networks functioning within the discussed inference engine. The knowledge contained in the fuzzy rules should be transferred into the structures of artificial neural network, where inference (also for new cases, not included in the knowledge base) would take place in accordance to its qualities. In order to realize this assumption, attention should be paid to proper preparation of cases used in the neural network learning process and its verification. Specific rules of knowledge base transformed into the form acceptable by the neural network constituted these cases. The module that uses characteristics of fuzzy sets, where the fuzzy scaling took place, was responsible for the transformation (understood as internal communication in the system). The number of cases for neural network was increased from initial 164 to 492 with the use of fuzzy sets characteristics. An inhouse method of "gravity centres relocation" was used here. This method consisted in relocation of gravity centres for fuzzy value, without changing it but simultaneously get a different sharp value after defuzzification (Fig. 2). Thus single-identical ranges were determined, within which gravity centres were relocated. There existed reasonable suspicions that initially small number of learning cases gives no guarantee that the process will succeed. These suspicions tuned out to be true and increasing the number of cases improved the process of neural network learning.

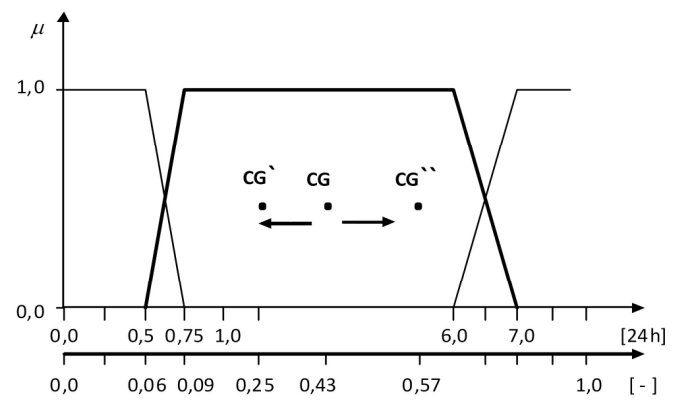

Figure 2. "Gravity centres relocation" method idea

In order to specify the predispositions of the chosen types of neural networks for the modelled problem (classification), a series of tests was performed with the following network types:

- multi-layers perceptron (MLP),

- radial basis function neural network (RBF),

- probabilistic neural network (PNN). 
Various architectures were built for these networks, they were also tuned (by the selection of learning factor, momentum, smoothing) and learning algorithms were chosen. Each time, prediction statistics (correct and incorrect), errors (related to learning, validation and testing) were determined and the errors' occurrence depending on learning progress (époques number) was observed. An example of error function (cross entropy) dependence from époques number for MLP networks is presented in (Fig. 3).

On the basis of performed analyses it was affirmed that the best solution is to use the network with a single output. Networks with 5 outputs, which embrace the structure of knowledge base "comprehensively", are not effective due to worse

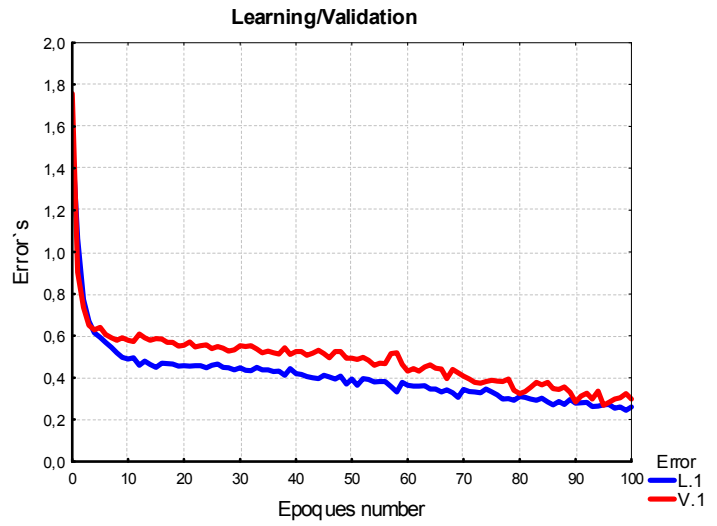

Figure 3. The diagram of the learning/validation of MLP network

results in correct predictions. Probably too small amount of learning cases and too many interneuronal connections make networks of such architecture have worse results than simpler networks, with one output (each time related to specific conclusion in the knowledge base). As far as networks' types are concerned, acceptable results were obtained for networks of PNN and MLP type (within the limited range). RBF networks did worse. An example of comparison is presented in Table 1.
Table 1. Example of comparison on network`s

\begin{tabular}{ccccc}
\hline ANN type & $\begin{array}{c}\text { Learn. } \\
\text { error }\end{array}$ & $\begin{array}{c}\text { Walid. } \\
\text { Terror }\end{array}$ & $\begin{array}{c}\text { Learn. } \\
\text { quality }\end{array}$ & $\begin{array}{c}\text { Walid. } \\
\text { quality }\end{array}$ \\
\hline $\begin{array}{c}\text { MLP } \\
\text { 7:14-10-1:1 }\end{array}$ & 0,0518 & 0,0631 & 0,9859 & 0,9818 \\
$\begin{array}{c}\text { MLP } \\
\text { 7:14-4-5:5 }\end{array}$ & 0,2484 & 0,2525 & 0,8593 & 0,8512 \\
RBF & 0,2982 & 0,3258 & 0,8861 & 0,8373 \\
$7: 14-37-1: 1$ & 0,2982 & & & \\
$\begin{array}{c}\text { PNN } \\
7: 14-246-2: 1\end{array}$ & 0,1463 & 0,1515 & 0,9796 & 0,9756 \\
\hline
\end{tabular}

Therefore, PNN networks, which had similarly acceptable results in all considered solutions, were selected for the purpose of inference engine functioning. On the basis of the networks' types that underwent the learning process relying on the cases that came from the knowledge base, the inference engine generates answers for new, real cases.

\section{HYBRIDITY IN THE SYSTEM}

The phenomenon of hybridity that exists in the system may be considered in two aspects. First of them is tool-hybridity and the other one methodichybridity when using and developing the system. Tool-hybridity consists in combining several intelligent techniques (artificial neural networks, fuzzy sets, knowledge base) within one tool, being also intelligent - advisory system. The phenomenon of tool-hybridity may be categorized as so called "immersing" - the formed neuro-fuzzy model submerged in the advisory system, co-working and communicating within. However taking into consideration the degree of mutual integration of particular intelligent modules (Fig. 4), hybrid model may be reckoned as loosely connected model, which allows for mutual communication via knowledge and data transfer. The whole works for one system and related problem, and the advisory system itself serves as a "clip".

Another hybridity level in the system is the methodical level connected with system usage and possibilities to update and develop it. Initially system functions relying on the knowledge base being a derivative of mental expert model. Assuming that the system is to be developed and used, it is possible to generate real observations of inputs and outputs. On that basis it is possible to create so called secon- 
dary knowledge base that relies on the observed actual parameters. The knowledge defined as primary and secondary may co-exist within the same base and further co-function in the inference engine. It is necessary, of course, to perform verification in order to exclude conflicting phenomena within one knowledge base.

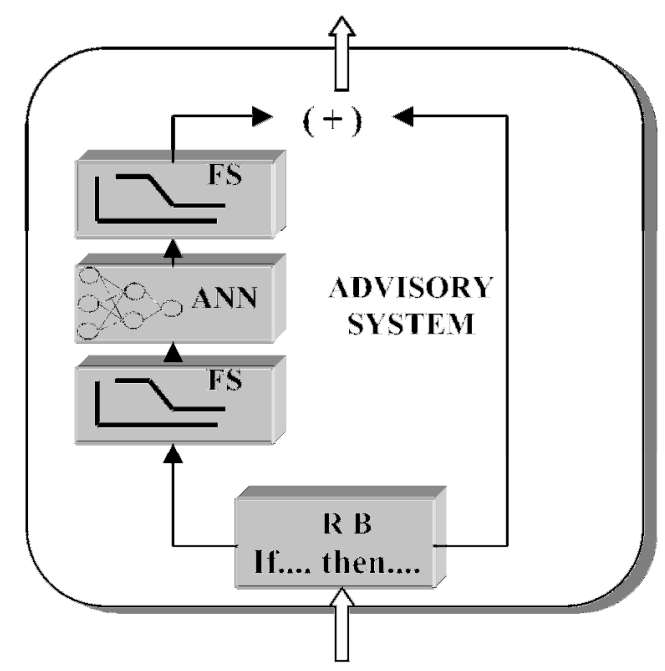

Figure 4. Tool-hybridity in advisory system

\section{PRACTICAL APPLICATION}

Presented advisory system underwent verification process. First type of verification was the verification of the system model itself and correctness in particular modules' functioning (knowledge base, inference engine, communication modules). The test of system's functioning with real cases was also a kind of verification. For some reasons the results of system's functioning have not been yet implemented in practice, however the modus operandi, dialog form and information gathered were already presented.

The damage of concrete industrial floor in the manufacturing shop in automotive industry (production of metal and rubber elements) constituted the considered situation (Fig. 5). The damage was fractures and scratches. An analysis for possible reasons was performed and possibilities of overloading the floor by functioning machines that were placed directly on the floor, with no foundations. On the basis of input data that result from the dialog formula an input vector was formed. It contained the information related to:

- type and depth of the damage - scratches, deep damages,

- mechanical loading - high loadings,

- thermal loading from $-\mathbf{2 0}^{\mathbf{0}}$ to $-\mathbf{3 5}^{\mathbf{0}} \mathbf{C}$,

- chemical loading - neutral interactions/ impacts,

- interactions/ impacts occurrence time and its character - constantly present,

- possibilities to exclude the facility or its part from the operation and related time of the repair - it is possible that the repair lasts up to 3 days,

- repair material application temperature $-\mathbf{2 0}^{\circ}$ $35^{\circ} \mathrm{C}$.

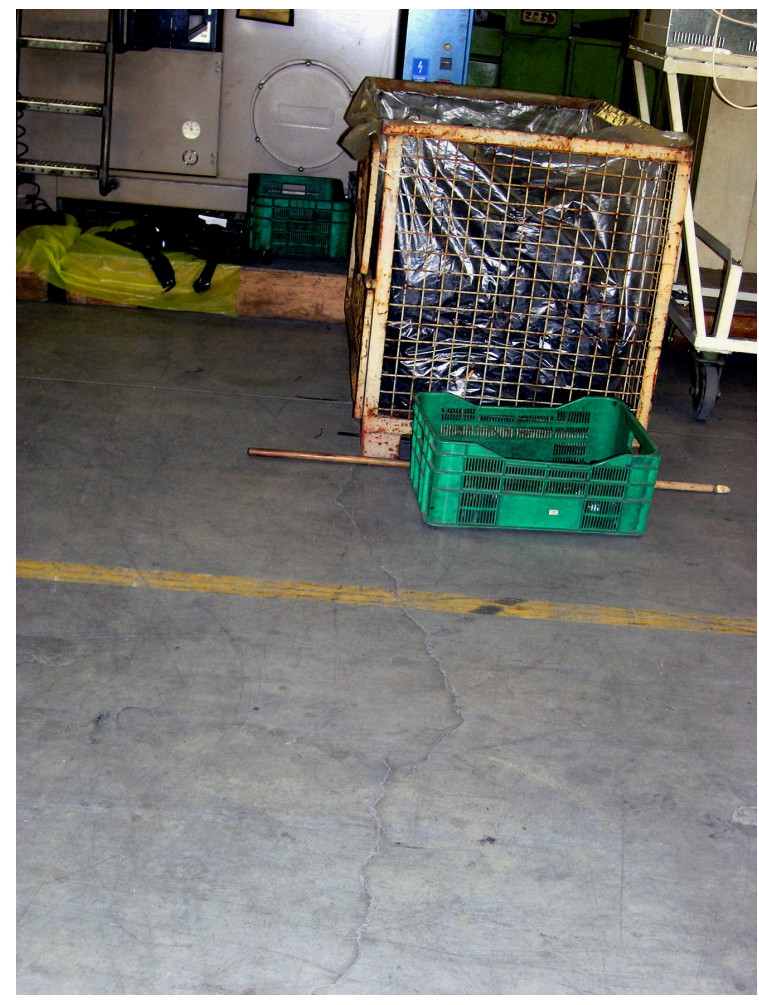

Figure 5. The damage of the industrial floor in the building 
On the basis of the obtained information connected to the above mentioned issues, an answer vector was created. This vector was then processed (by the operation of communication model related to fuzzy sets) and transformed into acceptable form by inference engine (1.0).

$\mathrm{X}[\mathrm{C} ; \mathrm{E} ; \mathrm{J} ; 0,416 ; 0,887 ; 0,650 ; 0,336]$

As a result of inference engine operation an output vector was created (2.0).

$\mathrm{Y}[0 ; 0 ; 1 ; 1 ; 1]$

This vector after another processing brought the system answers within possible solutions. Information from database related to the technology of specific solution, economic parameters and important information, as far the realization correctness is concerned, have been generated along with the output vector. The advisory system offered 3 possible solutions that met the requirements. A final solution should be sought within the field of these solutions, according to decision-maker's preferences (e.g. related to economic parameters). A human is responsible for taking such decision and the task of the advisory system is to generate the acceptable solutions with their description.

\section{CONCLUSIONS}

The presented hybrid advisory system may be considered as a successful tool within the scope of decision-taking support in the problem of repairs of industrial floors. Its operation meets the assumptions made while its development. The intelligent modules used in system's development process its correct operation both in the scope of mutual cooperation and communication, and within the scope of realization of special functions assigned to these modules. In order to further develop the existing advisory system it seems advisable to increase the system's resources (knowledge base and database development by adding new solutions and information), and also to use "data, text mining" techniques to accomplish that goal. The author suggests that the development and use of intelligent hybrid tools that support humans within the scope of repetitive problems will the issue of near future.
This paper sponsored as the researching project in years 2007-2009.

\section{REFERENCES}

[1] Kaplinski O. (2007) Methods and models of research in construction project engineering (in polish). Polish Sciennce Academy, Committee of Civil Engineering and Hydroengineering, Institute of Fundamental Technological Research. Warszawa

[2] Zavadskas E., Kaplinski O., Kaklauskas A., Brzezinski J. (1995) Expert systems in construction industry. Trends, potential \& applications. Technika. Vilnius.

[3] Chen J., Hsu S. (2007) Hybrid ANN-CBR model for disputed change orders in construction project. Automation in Construction, Vol. 17, No. 1. 56-64

[4] Khosrowshahi F., Howes R. (2005) A framework for strategic decision-making based on a hybrid decision support tools. The Electronic Journal of Information Technology in Construction. Vol. 10. 2005/9, 111124 http://www.itcon.org/2005/9

[5] Medsker L. M. (1994) Hybrid neural networks and expert systems. Kluwer Academic Publishers. Boston

[6] Pokojski J. (2005) Advisory systems in machines designing (in polish). WNT. Warszawa 